\title{
A Methodology for Designing Communication Protocols*
}

\author{
Gurdip Singh \\ Department of Computing and Information Sciences \\ 234 Nichols Hall, Kansas State University \\ Manhattan, Kansas 66506 \\ email: singh@cis.ksu.edu
}

\begin{abstract}
We propose a compositional technique for designing protocols. The technique involves specifying constraints between the events of the component protocols. A constraint may either require synchronization between certain events of the component protocols or may require inhibiting an event in one protocol on the occurrence of an event in another component protocol. We find both types of constraints useful in composing protocols. We demonstrate the applicability of the technique by deriving several protocols. The technique facilitates modular design and verification. Our technique, in conjunction with the sequential composition technique, can be used to design complex protocols.
\end{abstract}

\section{Introduction}

A communication protocol $P$ is a collection of processes, $P_{i}$, which execute concurrently and communicate via messages. Communication protocols typically perform several concurrent activities and modules for various activities may interact with one another in a complex way. The complexity of distributed protocols have led to compositional design and verification techniques in which protocols performing the various subfunctions are designed and verified separately and then integrated to form a more complex protocol. Several techniques have been proposed which use a protocol as the unit of modularity. These proposals involves the following three steps:

${ }^{*}$ This work was supported by NSF grant CCR9211621.

Permission to copy without fee all or part of this material is granted provided that the copies are not made or distributed for direct commercial advantage, the ACM copyright notice and the title of the publication and its date appear, and notice is given that copying is by permission of the Association of Computing Machinery. To copy otherwise, or to republish, requires a fee and/or specific permission.

SIGCOMM $94-8 / 94$ London England UK

(c) 1994 ACM 0-89791-682-4/94/0008..\$3.50
1. In the first step, a designer divides the functionality of a protocol into subfunctions.

2. In the second step, a protocol for each subfunction is obtained.

3. The third step involves combining the protocols to obtain a protocol for the entire problem.

The difficult part in combining the component protocols is specifying the interactions between the actions of these protocols. Different techniques use different forms of interactions such as imposing ordering on events, synchronizing events, imposing additional constraints by strengthening of guards, etc. In the sequentzal composition of protocols $P$ and $Q$, all events of $P_{i}$ are ordered before all events of $Q_{i}$. [CGL85] [CM83] [SR89] have proposed frameworks for sequential composition of protocols, in which a problem is divided into two or more subproblems which can be performed in sequence. Protocols solving the subproblems are designed and verified independently and then combined sequentially to obtain a protocol for the entire problem. [LT93] proposed an improved technique for sequential composition. In [Lin91], a variant of sequential composition was proposed in which protocols performed multiple functions but one function at a time (although which function to perform next is not known in advance).

In a parallel composition of protocols, the component protocols may execute concurrently (i.e., the events of $P_{i}$ and $Q_{i}$ may be interleaved). [BF88] proposed the notion of superimposition which is a parallel composition of a basic computation with some control protocol. The composition requires synchronizing the message-sending steps of the control protocol with those of the basic computation. A similar notion of superposition for UNITY 
specifications was proposed by [CM88]. The union construct proposed in [CM88] also allows interleaved execution of $P$ and $Q$ but does not impose any synchronization constraints. [Lin88] proposed a methodology of constructing a multi-function protocol by combining the protocols performing the various functions. The composition involves imposing operational constraints (by strengthening the guards of various actions) on the execution of the protocols solving the subproblems. [Sin93] proposed a technique which allows the component protocols to share variables as well as messages. This composition principle requires the component protocols to synchronize at events which update shared variables or send/receive common messages. [LT87] defined the composition of protocols specified as I/O automata in which the protocols interact with each other using input-output (i.e., output of one protocol may be the input for another protocol). [FLS87] used the $\mathrm{I} / \mathrm{O}$ automata model to give a modular correctness proof for a synchronizer. [Jon94] proposed a similar notion for I/O-systems in which components synchronize on communication events. Each technique essentially imposes constraints which restricts the execution of a component protocol.

In this paper, we propose a methodology for parallel composition of protocols. The composition involves executing the component protocols in an interleaved fashion subject to certain constraints. In our methodology, a constraint may either require synchronization between certain events of the component protocols or may require inhibiting an event in one protocol on the occurrence of an event in another component protocol. We find both types of constraints useful in composing protocols. Restricted forms of the synchronization constraints have been used in the formalisms discussed above (inhibitive constraint has not been used in these formalisms). We demonstrate the applicability of the technique by deriving several protocols. For example, for the transaction commitment problem, we first design a two-process protocol. We then obtain an N-process 3phase commit protocol by combining the two-process commit protocols using the synchronization constraint. By imposing inhibitive constraints on certain events of the same set of component protocols, we obtain the extended two-phase commit protocol. We also derive a data transfer protocol from simple component protocols by applying this technique. We give sufficient conditions to infer properties such as invariants, deadlockfreedom, unspecified receptions, and liveness properties of the composite protocol from those of the component protocols.

The compositional technique developed in this paper, together with the sequential composition technique, can be used to design complex communication protocols. It allows modular design and verification by allowing a designer to focus on one aspect at a time, and reuse of existing protocols. Different protocols can be designed from the same set of component protocols using different types of interactions. We also advocate the use of step-wise refinement techniques [LU90] [LT87] [WLL88] in conjunction with our compositional techniques. First, a simple protocol (maybe an inefficient one) can be derived using our technique. It could then be refined in a step-wise manner to optimize it or maybe more constraints can be added in order to satisfy certain additional properties.

This paper is organized as follows. In the next section, we present a formalism to specify protocols and discuss some examples. In Section 3 , we present our composition principle and derive some protocols. Section 3.1 describes rules to infer properties of the composite protocols. In Section 4, we discuss a derivation of a data transfer protocol. Section 5 concludes the paper.

\section{Model}

A distributed protocol $P$ is a set of processes $\left[P_{1}\left\|P_{2}\right\|\right.$ $\left.\cdots \| P_{n}\right]$. Each $P_{i}$ is of the form:

$$
*\left[\square_{l=1}^{m_{1}} \text { en }\left(a_{l}\right) \longrightarrow a_{l}:<s_{l}>\right]
$$

where $a_{l}$ is the label of the action, $e n\left(a_{l}\right)$ is the enabling condition (which is a boolean expression) and $s_{l}$ is the computation associated with the action. A communication channel from $P_{i}$ to $P_{j}$ is modeled as a sequence $c h_{i, j}$ of messages. The statement $j ! M\left(\right.$ arg $\_$list $)$executed by $P_{i}$ causes $M\left(\arg \_\right.$list $)$to be appended to the end of $c h_{i, j}$, and the statement $i$ ? $M$ (para_list) executed by $P_{j}$ removes the message at the head of $c h_{i, j}$ and stores the contents of the message in para_list. Let variable $\left(P_{i}\right)$ denote the set of variables which appear in $P_{i}$ (except the variables modeling the channels) and variable $(P)=$ variable $\left(P_{1}\right) \cup \ldots \cup$ variable $\left(P_{n}\right)$. Let mess $\left(P_{i}\right)$ denote the set of messages sent or received by $P_{i}$ and mess $(P)$ $=$ mess $\left(P_{1}\right) \cup \ldots \cup$ mess $\left(P_{n}\right)$ (we assume that messages with the same name sent over different links are different; for this purpose, we will assume that each message is indexed with the channel over which it is sent). 


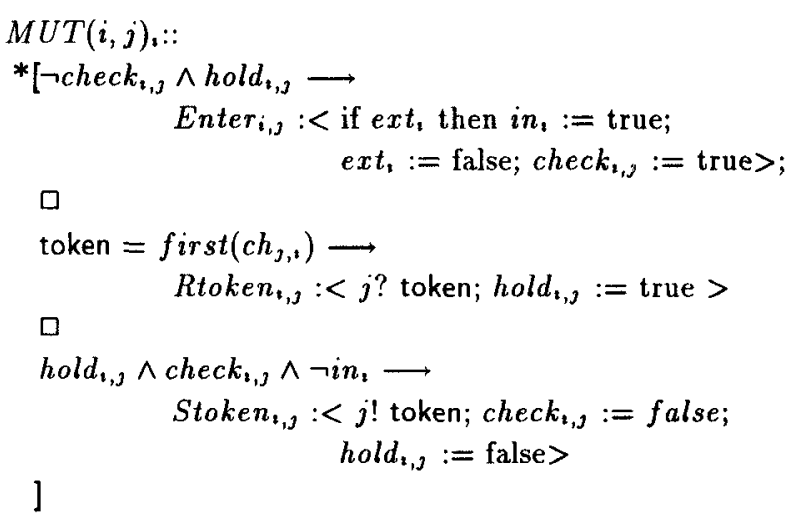

Figure 1: Protocol $M U T(i, j)$

Figure 1 gives a two-process mutual exclusion protocol, $\operatorname{MUT}(i, j)$, for processes $i$ and $j$. Initially, $i n_{i}$ $=i n_{j}=$ false and $c h_{i, j}=c h_{j, i}=\lambda$. Furthermore, one of hold hi,j $_{j}$ and hold h $_{j, i}$ is true, and check $_{i, j}=$ false if hold $_{i, j}=$ false initially. We assume that $e^{x} t_{i}$ is an external variable which is set to true if $i$ wants to enter the critical section and $i n_{i}$ is set to false when $i$ exits the critical section. If $i$ has the token, it can enter the critical section. On exiting the critical section, it sends the token to $j$.

The state of $P_{i}$ is defined by the values of variables in variable $\left(P_{i}\right)$. The state of a channel from $P_{i}$ to $P_{j}$ is the value of $c h_{i, j}$. The state of the system is a tuple $\left(s_{1}, \ldots, s_{n}, c h_{1,1}, c h_{1,2} \ldots, c h_{n-1, n}\right)$, where $s_{i}$ 's represent the states of the processes and $c h_{i, j}$ 's represent the states of the channels. An event is specified by an action and its enabling condition. An event occurs when the computation associated with its action is executed. An execution of a protocol is a maximal sequence, $g_{0} \rightarrow_{e_{0}} g_{1} \rightarrow_{e_{1}} \cdots$, such that $g_{0}$ is the initial system state, $e n\left(e_{l}\right)$ is true in state $g_{l}$ and the execution of the computation associated with $e_{l}$ transforms state $g_{l}$ to $g_{l+1}$. We say that $g$ is reachable from $g^{\prime}$ if there exists a sequence of events which takes the system from state $g^{\prime}$ to $g$. Let $a^{2}$ denote the event corresponding to the $i^{\text {th }}$ execution of action $a$. For any two actions $a$ and $b$ of $P, a<_{p} b$ if in each execution $e x$ of $P, a^{i}$ precedes $b^{2}$ and $b^{i}$ precedes $a^{i+1}$ (we will omit the subscript in $<_{p}$ whenever it is clear from the context). For example, in $\operatorname{MUT}(i, j)$, Stoken $i, j<$ Rtoken $_{i, j}$ if $i$ has the token initially. We say that $a$ is the final action of $P_{i}$ if in all executions of $P$, no action of $P_{i}$ other than $a$ is enabled after the occurrence of $a$.
An assertion $I$ is an invariant if $I$ is true in the initial state and in all states reachable from the initial state. For example, $\left(i n_{i} \Rightarrow \neg i n_{j}\right) \wedge\left(i n_{j} \Rightarrow \neg i n_{i}\right)$ is an invariant of $M U T(i, j)$. Protocol $P$ is free from unspecified receptions if the following is true: for each reachable state $s$, if $m=f i r s t\left(c h_{i, j}\right)$ in state $s$ then there exists an action $a$ in $P$, which receives $m$ such that $e n(a)$ is true in $s$. For example, $M U T(i, j)$ is free from unspecified receptions since a message can be received in any state. Protocol $P$ is subject to deadlocks if there exists a state $s$ such that it has not terminated and no action is enabled at any process in state $s$.

We assume that each protocol system satisfies the following strong fairness condition: If an action is enabled infinitely often then its execution cannot be delayed indefinitely. We will now define the $\sim$ relation. $\alpha \sim \beta$ in an execution iff the following holds: For all $x$, if $\alpha$ is true in $g_{x}$, then there exists a state $g_{y}, y \geq x$, such that $\beta$ holds in $g_{y} . \alpha \leadsto \beta$ in $P$ if $\alpha \sim \beta$ in all executions of $P$. Informally, $\alpha \sim \beta$ implies that if $\alpha$ becomes true then $\beta$ will become true in the future. For example, $e x t_{i} \leadsto i n_{i}$ in $M U T(i, j)$ implies if $i$ wants to enter the critical section, it will eventually do so.

We now give the proof rules to infer liveness properties. In the following, by $\{\alpha\} a\{\beta\}$, we will mean that if $\alpha$ is true before the execution of $a$ then $\beta$ is true after the execution of $a$. Following [LU90], $\alpha \sim \beta$ via $a_{p}$ in $P$ if the following holds:

(1) $\alpha \Rightarrow e n\left(a_{p}\right)$ is an invariant of $P$

(2) $\{\alpha\} a_{p}\{\beta\}$, and

(3) for all actions $b_{p},\left\{\alpha \wedge e n\left(b_{p}\right)\right\} b_{p}\{\alpha \vee \beta\}$.

If $P$ is free from unspecified receptions then for any message $m,\left(m \in c h_{i, j}\right) \sim\left(m=f i r s t\left(c h_{i, j}\right)\right)$. Further, $\alpha \sim \beta$ in $P$ if one of the following is true:

(L1) $\alpha \sim \beta$ via $a_{p}$

(L2) $\alpha \Rightarrow \beta$ is an invariant

(L3) $\alpha \leadsto \gamma$ and $\gamma \sim \beta$

(L4) $\alpha \wedge$ bool $\sim \beta$ and $\alpha \wedge \neg$ bool $\sim \beta$.

\section{A Compositional Approach}

In this section, we present the composition principle. We will consider composition of two component protocols only (the principle can be extended to any number of component protocols). Let $P \equiv\left[P_{1}\|\cdots\| P_{n}\right]$ and $Q \equiv\left[Q_{1}\|\cdots\| Q_{n}\right]$ be the two component protocols. We will obtain a composite protocol $R$, denoted by $\operatorname{Comp}(P, Q)$. The composite protocol $R$ involves a 


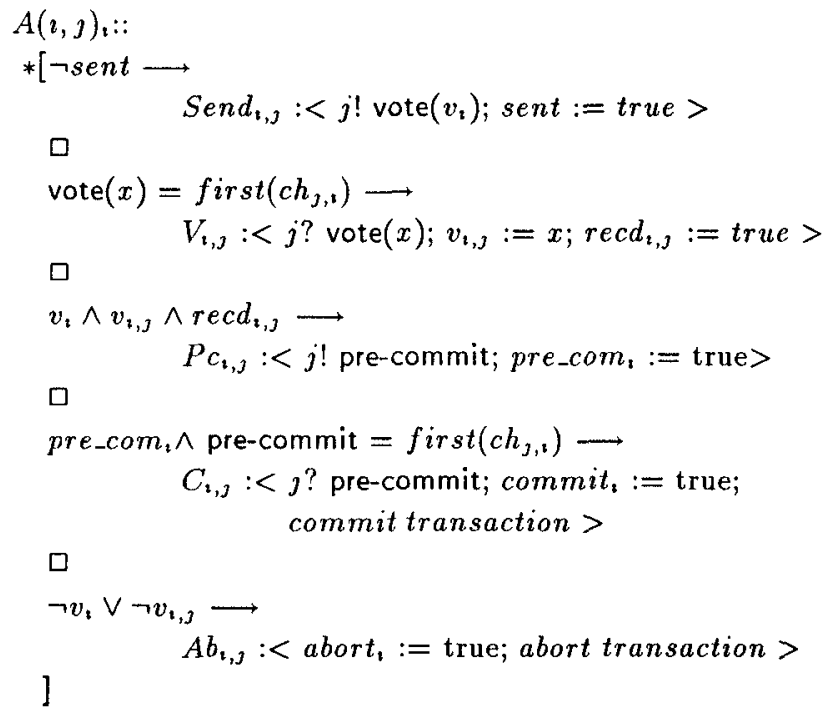

Figure 2: Two process Commit Protocol

concurrent execution of both $P$ and $Q$ subject to some constraints. In the following, we will use $a_{p}, b_{p}, \ldots$ to denote actions of $P$ and $a_{q}, b_{q}, \ldots$ to denote actions of $Q$. We will now explain the various constraints using examples:

- Synchronization constraint: A synchronization constraint between actions $a_{p}$ and $a_{q}$ requires that their execution be synchronized i.e., the protocol must reach a state in which both $e n\left(a_{p}\right)$ and $e n\left(a_{q}\right)$ are true and then execute both actions. For example, consider the two-process commit protocol $A(i, j)$, for processes $i$ and $j$, given in Figure 2 (the protocol is given for process $i$; process $j$ is symmetric). Each process sends its vote to the other process. If both $i$ and $j$ have voted positively then both send a pre-commit message to each other. On receiving a pre-commit message, $i$ commits the transaction (the protocol corresponds to normal phase; recovery and timeout actions are not considered). In the three phase commit protocol, (c1) a process pre-commits if all processes have voted positively and (c2) a process commits if it knows that every process knows that all other processes have voted positively (i.e, it must know that all processes have pre-committed). We can obtain a 3-process three-phase commit protocol, $A 3 P(i, j, k)$, from protocols $A(i, j), A(i, k)$ and $A(k, j)$ by synchronizing the execution of following pairs of actions at process $i$ : $\left(\operatorname{Send}_{i, j}\right.$, Send $\left._{i, k}\right),\left(P c_{i, j}, P c_{i, k}\right)$ and $\left(C_{i, j}, C_{i, k}\right)$ (similar synchronization is carried out at other processes). For example, the synchronization of actions

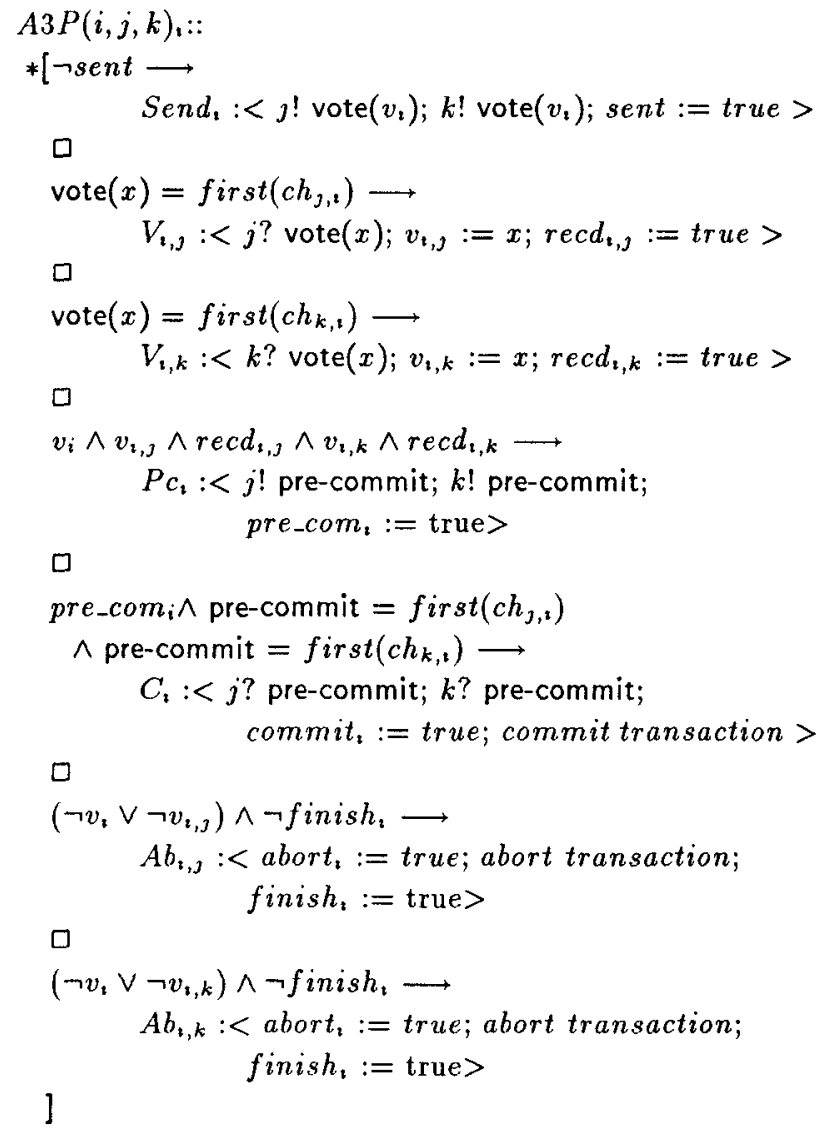

Figure 3: Three-phase Commit Protocol

$\left(P c_{i, j}, P c_{i, k}\right)$ ensures (c1) (since it will require $i$ to wait until both $j$ and $k$ have voted positively) and synchronization of $\left(C_{i, \jmath}, C_{i, k}\right)$ ensures (c2) (this will require that $i$ commits after it has received a pre-commit message from both $j$ and $k$ ). The final protocol is shown in Figure 3 (the derivation is discussed later). Similarly, we can combine two-process mutual exclusion protocols (of Figure 1), one for each pair of processes, to obtain N-process mutual exclusion protocol by synchronizing Enter ${ }_{i, k}$ for all $k$ at process $i$. This constraint can also be used to model superimposition and the type of compositions discussed in [Lin88].

- Inhibitive constraint: In some compositions, the occurrence of an event in one protocol may require inhibition of events in the other component protocol. For example, consider again the commit protocol shown in Figure 2. In the extended two-phase commit protocol, (d1) a process pre-commits if it knows that all processes have voted positively and $(\mathrm{d} 2)$ a process commits after it knows that at least one other process knows all the votes. In this case, $i$ will commit the 
transaction on receiving a pre-commit message from either $j$ or $k$. If the message is received from $j$ first then the transaction is committed. When the pre-commit message from $k$ is received later, it is ignored (the transaction is committed only once). We refer to such an constraint as inhibitive constraint between events $C_{i, j}$ and $C_{i, k}$, which ensures (d2). Intuitively, an inhibitive constraint between $a_{p}$ and $a_{q}$ specifies that only one of $a_{p}$ and $a_{q}$ be executed (whichever occurs first). The combined protocol is shown in Figure 4 and its derivation is discussed later.

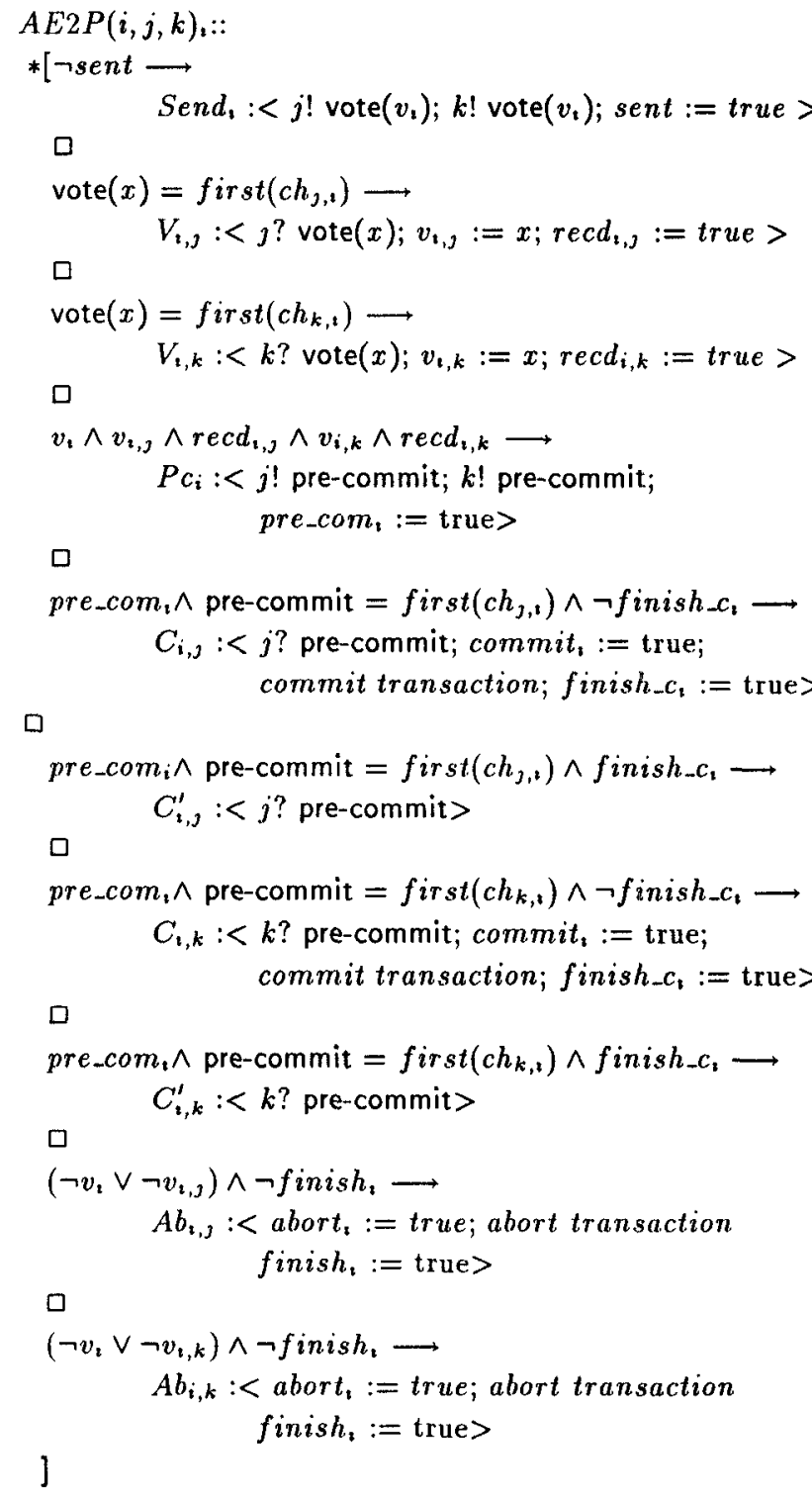

Figure 4: Extended Two-phase Commit Protocol

As another example, consider a simple protocol, $D^{\{i, j\}}$, in which process $i$ sends some information to process $j$ in a message data. On receiving the message data, process $j$ performs the action $R e c_{j}$ which involves storing the information and incrementing a counter. Assuming that the channel is perfect, this protocol performs the required function. However, if the link between $i$ and $j$ may lose messages, then $i$ may have to retransmit the information. Intuitively, this corresponds to initiating another copy of $D^{\{i, j\}}$. Thus, $i$ may initiate several copies of this protocol which execute concurrently. Process $j$ waits for the information to arrive in a message corresponding to any one of the concurrently initiated copies of the protocol. On receiving the message data for the first time (corresponding to any copy of the protocol), it performs the action $R e c_{j}$ and then inhibits the execution of $R e c_{j}$ in other copies of the protocol (i.e., on receiving subsequent data message, it ignores them). This can be viewed as an inhibitive constraint between action $R e c_{j}$ in the different copies of $D^{\{i, j\}}$.

Although the interactions discussed above are simple, we will show that complex protocols can be derived by hierarchical application of this composition principle. In Section 4 , we obtain a data transfer protocol using this technique.

To preserve the properties of the component protocols, we have to impose certain restrictions on the interactions. In the following, we will formally define the composition and the restrictions. We first define the notion of matching actions. Actions $a_{1}:\left\langle s ; s_{1}>\right.$ and $a_{2}:<s ; s_{2}>$ of $P_{i}$ and $Q_{i}$ respectively are matching actions if $s_{1}$ and $s_{2}$ do not refer to any variable in variable $\left(P_{i}\right) \cap$ variable $\left(Q_{i}\right)$ or send/receive a message in mess $\left(P_{i}\right) \cap \operatorname{mess}\left(Q_{i}\right)$ (similarly, $a_{1}:\left\langle s_{1} ; s>\right.$ and $a_{2}:\left\langle s_{2} ; s\right\rangle$ are also matching). For example, the actions

$$
\begin{aligned}
& \text { Send }_{i, j}:<j ! \text { request; sent }:=\text { true }> \\
& \text { Send }_{i, k}:<k ! \text { request; sent }:=\text { true }>
\end{aligned}
$$

in $A(i, j)$ and $A(i, k)$ respectively are matching, where $s=$ 'sent $:=$ true' (note that although the message request is sent in both protocols, it is sent over different links and therefore, is not considered a common message). Let shared $(P, Q)$ denote the set of actions in $P$ and $Q$ which either update a variable in variable $(P) \cap$ variable $(Q)$ or send a message in mess $(P) \cap \operatorname{mess}(Q)$. We say that $P$ and $Q$ are matching protocols if for each action $a$ in shared $(P, Q)$ which belongs to $P(Q)$, there exists a matching action in $Q(P)$. For example, protocols $A(i, j)$ and $A(i, k)$ are matching since shared $(A(i, j), A(i, k))=$ 


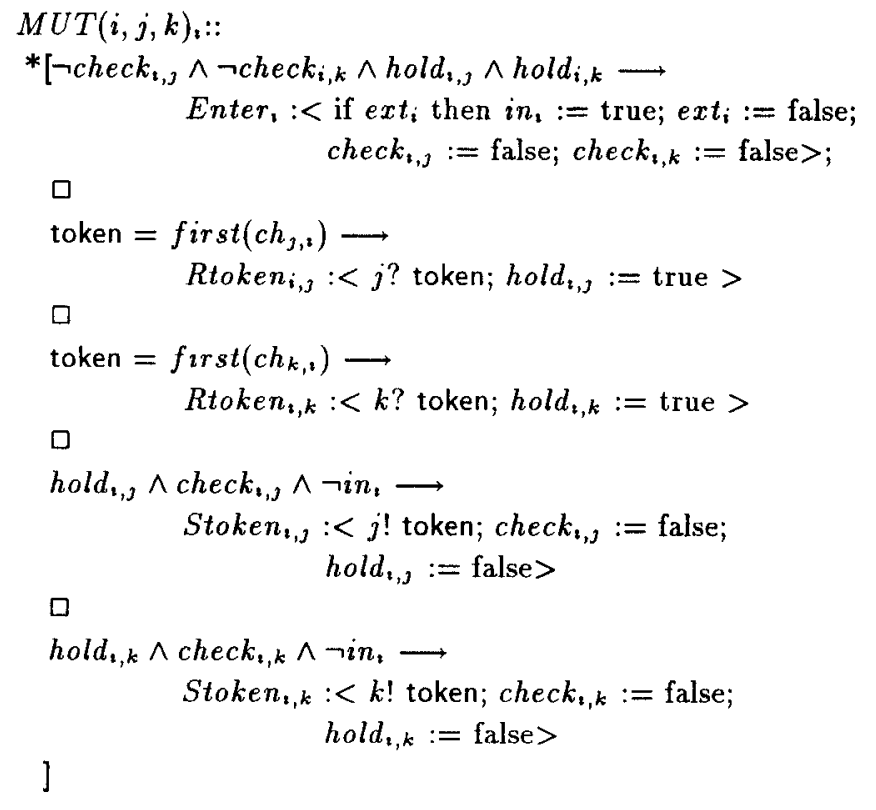

Figure 5: Protocol $M U T(i, j, k)$

$\left\{\operatorname{Send}_{i, j}, P c_{i, j}, C_{i, j}\right.$, Send $\left._{i, k}, C_{i, k}, P c_{i, k}\right\}$, and $\left(\right.$ Send $_{i, j}$, Send $\left._{i, k}\right),\left(P c_{i, j}, P c_{i, k}\right)$ and $\left(C_{i, j}, C_{i, k}\right)$ are matching pairs of actions (actions for only process $i$ are shown; similar matching actions exist at other processes)

Let $P$ and $Q$ be matching protocols. The composition is performed with respect to two sets, $\operatorname{synch}(P, Q)$ and inhibit $(P, Q)$, which consists of matching pairs $\left(a_{p}, a_{q}\right)$ of actions (first action belongs to $P$ and the second belongs to $Q)$. We say that action $a$ is in $\operatorname{synch}(P, Q)(\operatorname{inhibit}(P, Q))$ if it is a component of at least one pair in $\operatorname{synch}(P, Q)(\operatorname{inh} i b i t(P, Q))$. We require each action in shared $(P, Q)$ to be present in either $\operatorname{synch}(P, Q)$ or inhibit $(P, Q)$ (but not both). This ensures that during the concurrent execution of $P$ and $Q$, shared variables are updated only when the update is consistent with both component protocols. We allow non-shared actions to be in $\operatorname{synch}(P, Q)$ and $\operatorname{inhibit}(P, Q)$ because in some compositions, we have to impose some ordering between non-shared actions. We will now give the rules to obtain the composite protocol $R=\operatorname{Comp}(P, Q)$. The set of guarded actions of $R_{i}$ is obtained as follows:

- If $a:\langle s\rangle$ is not in $\operatorname{synch}(P, Q) \cup \operatorname{inhibit}(P, Q)$ then $e n(a) \longrightarrow a:<s>$ is in $R_{i}$.

- If $a:<s ; s_{a}>$ and $b:<s, s_{b}>$ is a matching pair in $\operatorname{synch}(P, Q)$, then $e n(c) \longrightarrow c:<s ; s_{a} ; s_{b}>$ is in
$R_{i}$, where $c$ is a new label and $e n(c)=e n(a) \wedge e n(b)$. This rule ensures the synchronized execution of $a$ and $b$.

- If $a:\left\langle s ; s_{a}>\right.$ and $b:<s, s_{b}>$ is a matching pair in $\operatorname{inhibit}(P, Q)$, the following actions are in $R_{i}$ : en $(a) \wedge \neg$ new $\longrightarrow a:\left\langle s, s_{a}\right.$; new $:=$ true $>$; en $(a) \wedge$ new $\longrightarrow a^{\prime}:<s_{a}>$; en(b) $\wedge \neg$ new $\longrightarrow b:<s, s_{b}$; new $:=$ true $>$; $e n(b) \wedge n e w \longrightarrow b^{\prime}: s_{b}>$, where new is a new boolean variable which is initially false, and $a^{\prime}$ and $b^{\prime}$ are new labels. Action $a^{\prime}$ $\left(b^{\prime}\right)$ represents the non-shared part of $a(b)$. Action $a^{\prime}\left(b^{\prime}\right)$ will be executed only if $b(a)$ has already occurred (in this case, the shared part $s$ of the action has already been executed by $b(a)$ ).

We will derive a three process mutual exclusion protocol using the protocol of Figure 1. It can be seen that $\operatorname{MUT}(i, j)$ and $\operatorname{MUT}(i, k)$ are matching. Hence, we can obtain $M 1=\operatorname{Comp}(M U T(i, j), M U T(i, k))$ with $\operatorname{synch}(\operatorname{MUT}(i, j), \operatorname{MUT}(i, k))$

$\left\{\left(\right.\right.$ Enter $_{i, j}$, Enter $\left.\left._{i, k}\right)\right\}$. Further, $M 1$ and $\operatorname{MUT}(j, k)$ are also matching. We combine these protocols with $\operatorname{synch}(M 1, M U T(j, k))$

$=\left\{\left(\right.\right.$ Enter $_{j, k}$, Enter $\left._{j, i}\right),\left(\right.$ Enter $_{k, j}$, Enter $\left.\left._{k, i}\right)\right\}$ to obtain $\operatorname{MUT}(i, j, k)$, which is shown in Figure 5.

By specifying different synch and inhibit sets for the same component protocols, we can obtain different composite protocols. We will now obtain the commit protocols discussed previously. $A(i, j)$ and $A(i, k)$ are matching protocols. Hence, we can obtain $A 3 P^{\prime}$ by combining them using (the sets are shown for process $i$ only):

$$
\begin{aligned}
& \operatorname{synch}(A(i, j), A(i, k))= \\
& \quad\left\{\left(\operatorname{Send}_{i, j}, \operatorname{Send}_{i, k}\right),\left(P c_{i, j}, P c_{i, k}\right),\left(C_{i, j}, C_{i, k}\right)\right\}
\end{aligned}
$$$$
\operatorname{inhibit}(A(i, j), A(i, k))=\left\{\left(A b_{i, j}, A b_{i, k}\right)\right\} \text {. }
$$

We find that $A 3 P^{\prime}$ and $A(j, k)$ are also matching. We can combine these protocols using (the sets are shown for process $j$ only; constraints for $k$ are similar):

$$
\begin{aligned}
& \operatorname{synch}\left(A 3 P^{\prime}, A(j, k)\right)= \\
& \quad\left\{\left(\operatorname{Send}_{j, i}, \operatorname{Send}_{j, k}\right),\left(P c_{j, i}, P c_{j, k}\right),\left(C_{j, i}, C_{j, k}\right)\right\} \\
& \text { inhibit }\left(A 3 P^{\prime}, A(j, k)\right)=\left\{\left(A b_{j, i}, A b_{j, k}\right)\right\} .
\end{aligned}
$$

The final protocol, $A 3 P(i, j, k)$, is shown in Figure 3 . Similarly, the protocol of Figure 4 is obtained as follows: We first obtain $A E 2 P^{\prime}$ by combining $A(i, j)$ and $A(i, k)$ using (the sets are shown for process $i$ only): $\operatorname{synch}(A(i, j), A(i, k))=$

$\left\{\left(\operatorname{Send}_{i, j}, \operatorname{Send}_{i, k}\right),\left(P c_{i, j}, P c_{i, k}\right)\right\}$

$\operatorname{inhibit}(A(i, j), A(i, k))=\left\{\left(C_{i, j}, C_{i, k}\right),\left(A b_{i, j}, A b_{i, k}\right)\right\}$. We find that $A E 2 P^{\prime}$ and $A(j, k)$ are also matching. We 
combine these protocols using (the sets are shown for process $j$ only; constraints for $k$ are similar):

$$
\begin{aligned}
& \operatorname{synch}\left(A E 2 P^{\prime}, A(j, k)\right)= \\
& \left\{\left(\operatorname{Send}_{j, i}, \operatorname{Send}_{j, k}\right),\left(P c_{j, i}, P c_{j, k}\right)\right\} \\
& \text { inhibit }\left(A E 2 P^{\prime}, A(j, k)\right)= \\
& \left\{\left(C_{j, i}, C_{j, k}\right),\left(A b_{j, i}, A b_{j, k}\right)\right\} .
\end{aligned}
$$

As discussed in the introductory section, once a protocol is obtained using our technique, a step-wise refinement method may be employed to simplify or optimize the protocol. For example, we can simplify $A E 2 P(i, j, k)$ of Figure 4 by combining the last two guarded commands into a single one.

\subsection{Properties of the Composite Proto- cols}

In this section, we present results which enable us to infer properties of the composite protocol from those of the component protocols (due to space constraints, the proofs of the theorems are not presented here).

\subsubsection{Invariant Properties}

In general, a composition may involve both synchronization and inhibitive constraints. Synchronization constraints only impose restrictions on a component protocol $P$ and may restrict its behavior. However, no updates to variables in variable $(P)$ are made which are inconsistent with $P$ (i.e., only those updates which are allowed by $P$ are made). This however may not be the case with inhibitive constraints. For example, in $A E 2 P(i, j, k), C_{i, j}$ and $C_{i, k}$ are combined inhibitively. Consider the case in which $C_{i, j}$ occurs before $C_{i, k}$ in an execution ex of $A E 2 P(i, j, k)$. Then, in ex, the transaction is committed earlier than it would have if $A(i, k)$ was executed alone. This could invalidate some invariants of $A(i, k)$. We require that each component protocol $P$ satisfy the following conditions for inhibitively composed events:

- Let $a_{p} \in$ inhibit $(P, Q)$ be an action in $P_{i}$. Then (1) $a_{p}$ is a final action of the protocol, (2) for any other action $b_{p}$ of $P_{i}$, if $b_{p}$ immediately precedes $a_{p}$ in an execution then $b_{p} \in \operatorname{synch}(P, Q)$, and (3) $a_{p}$ does not involve receiving a shared message.

Condition (3) is a natural one since inhibitive constraint is imposed on events which perform the same action but via different methods (which will involve different sets of messages). We require (1) and (2) to simplify the proof rules. In the full paper, we have given conditions to remove (1) and (2) ${ }^{1}$. These conditions are satisfied by protocols discussed in the paper. We have the following theorems:

Theorem 3.1 Let $I$ be an invariant of $P$ (Q) which refers only to variables in variable $(P)$ (variable $(Q))$. If $I$ does not refer to variables updated by actions in inhibit $(P, Q)$ then $I$ is an invariant of $R$.

Theorem 3.2 Let $R=\operatorname{Comp}(P, Q)$. If $I$ is an invariant of both $P$ and $Q$ which refers to variables in variable $(P) \cap$ variable $(Q)$ then $I$ is an invariant of $R$.

For the mutual exclusion protocol of Figure 5, no action is subject to inhibitive constraints (hence, the condition of Theorem 3.1 that $I$ does not refer to variables updated by actions in $\operatorname{inhibit}(P, Q)$ is trivially satisfied). Since $i n_{i} \Rightarrow \neg i n_{j}$ and $i n_{i} \Rightarrow \neg i n_{k}$ are invariants of $\operatorname{MUT}(i, j)$ and $M U T(i, k)$ respectively, we can conclude that $\left(\left(i n_{i} \Rightarrow \neg i n_{j}\right) \wedge\left(i n_{i} \Rightarrow \neg i n_{k}\right)\right)$ is an invariant of $\operatorname{MUT}(i, j, k)$. Similarly, commit ${ }_{j} \Rightarrow$ vote $_{j} \wedge$ vote $_{k}$ is an invariant of both $A E 2 P^{\prime}$ and $A(j, k)$ respectively. Hence, it is an invariant of $A E 2 P(i, j, k)$ (from Theorem 3.2).

Finally, some invariant properties may be inferred due to the constraints. Let occur $(e)$ denote the fact that $e$ has occurred. If $\left(a_{p}, a_{q}\right) \in \operatorname{synch}(P, Q)$ then we know that the $i^{\text {th }}$ execution of $a_{p}$ is synchronized with the $i^{t h}$ execution of $a_{q}$. Hence, we have the following theorem.

Theorem 3.3 If $\left(a_{p}, a_{q}\right)$ are matching actions in $\operatorname{synch}(P, Q)$ then for any $i>0, \quad$ en $\left(a_{p}^{i}\right) \Rightarrow \operatorname{occur}\left(a_{q}^{i-1}\right)$ and en $\left(a_{q}^{i}\right) \Rightarrow \operatorname{occur}\left(a_{p}^{i-1}\right)$ invariants of $R$.

\subsubsection{Unspecified Reception}

We will now consider the property of unspecified reception. Let $\left(a_{p}, a_{q}\right)$ belong to $\operatorname{synch}(P, Q)$. If $a:\left\langle s ; s_{a}\right\rangle$ and $b:<s ; s_{b}>$ both involve receiving a non-shared message, say msg1 and msg2 respectively, then in $R$, msg 1 can be received only if $\mathrm{msg} 2$ has also arrived and vice-versa. This might lead to a situation with unspecified reception (if msg1 has arrived and msg 2 has not). Therefore, we will deal with compositions in which the

\footnotetext{
${ }^{1}$ For example, if we want to impose inhibitive constraints on an action of $P$ which is not a final action, then in some cases, we may be able to decompose $P$ into two sequential components, $P 1$ and $P_{2}$, so that $P_{1}$ satisfies (1). $P_{1}$ can be combined with $Q$ and the resulting composite protocol can be combined with $P 2$ using sequential composition.
} 
actions being combined synchronously do not involve receiving a non-shared message. Note that in some cases, if $s$ involves receiving a message, we can modify $P$ by splitting $a$ into two actions, $a_{1}:\left\langle s_{a}\right\rangle$ and $a_{2}:\langle s\rangle$ with a constraint that $a_{2}$ be executed after occurrence of $a_{1}$. Let $\left(a_{p}, a_{q}\right) \in \operatorname{inhibit}(P, Q)$. In this case, we do not want the occurrence of $a_{p}$ to disable the execution of $a_{q}^{\prime}$. Hence, we require that both en $\left(a_{p}\right)$ and $e n\left(a_{q}\right)$ do not refer to any shared variable updated by $a_{p}$ (or $a_{q}$ ) (in the respective component protocols). With these restrictions, we have the following theorem:

Theorem 3.4 If both $P$ and $Q$ are free from unspecified receptions then $R$ is free from unspecified reception.

\subsubsection{Deadlock Freedom}

In general, $R$ may be subject to deadlock even though both $P$ and $Q$ are deadlock free. Deadlocks may be caused due to the synchronization constraints or due to the fact that $R$ has unspecified receptions. For example, let $\operatorname{synch}(P, Q)=\left\{\left(a_{p}, a_{q}\right),\left(b_{p}, b_{q}\right)\right\}$. If $a_{p}<_{p} b_{p}$ and $b_{q}<_{q} a_{q}$ then the composite protocol will deadlock. Thus, if the ordering between $a_{p}$ and $b_{p}$ conflicts with that between $a_{q}$ and $b_{q}$, there is a potential for deadlock. In the following, we will give a sufficient condition to infer that there exist no deadlocks due to the synchronization constraints.

We say that $\left(a_{p}, b_{p}\right)$ dominates $\left(a_{q}, b_{q}\right)$ iff the following hold: (1) if $b_{q}^{i}$ awaits the occurrence of $a_{q}^{j}$ in any execution of $Q$ then $b_{p}^{i}$ awaits accurrence of $a_{p}^{j}$ in all executions of $P$, and (2) if $a_{q}^{2}$ awaits the occurrence of $b_{q}^{j}$ in any execution of $Q$ then $a_{p}^{i}$ awaits occurrence of $b_{p}^{\gamma}$ in all executions of $P$. Informally, if $\left(a_{p}, b_{p}\right)$ dominates $\left(a_{q}, b_{q}\right)$ then the ordering between $\left(a_{p}, b_{p}\right)$ is at least as strict as the ordering between $\left(a_{q}, b_{q}\right)$, and therefore, the ordering between $a_{q}$ and $b_{q}$ will not conflict with the ordering between $a_{p}$ and $b_{p}$. For example, $\left(\right.$ Enter $_{\jmath, k}$, Enter $\left._{k, j}\right)$ dominates $\left(\right.$ Enter $_{j, \imath}$, Enter $\left._{k, i}\right)$ because Enter $_{j, k}<$ Enter $_{k, j}$ while the Enter $r_{j, 2}^{x}$ and Enter ${ }_{k, i}^{x}$ are concurrent (Enter ${ }_{j, i}^{x}$ must await the occurrence of Enter $r_{k, i}^{x-1}$ and vice-versa; but Enter ${ }_{j, k}<$ Enter $_{k, j}$ implies that Enter $_{j, k}$ must await the occurrence of Enter $k, j$ and vice-versa). Protocol $P$ dominates $Q$ if for each pair of matching actions $\left(a_{p}, a_{q}\right)$ and $\left(b_{p}, b_{q}\right),\left(a_{p}, b_{p}\right)$ dominates $\left(a_{q}, b_{q}\right)$. We will now give a sufficient condition for deadlock freedom:

Theorem 3.5 $R$ is deadlock free if (1) $P$ and $Q$ are deadlock free, (2) both $P$ and $Q$ are free from unspecified

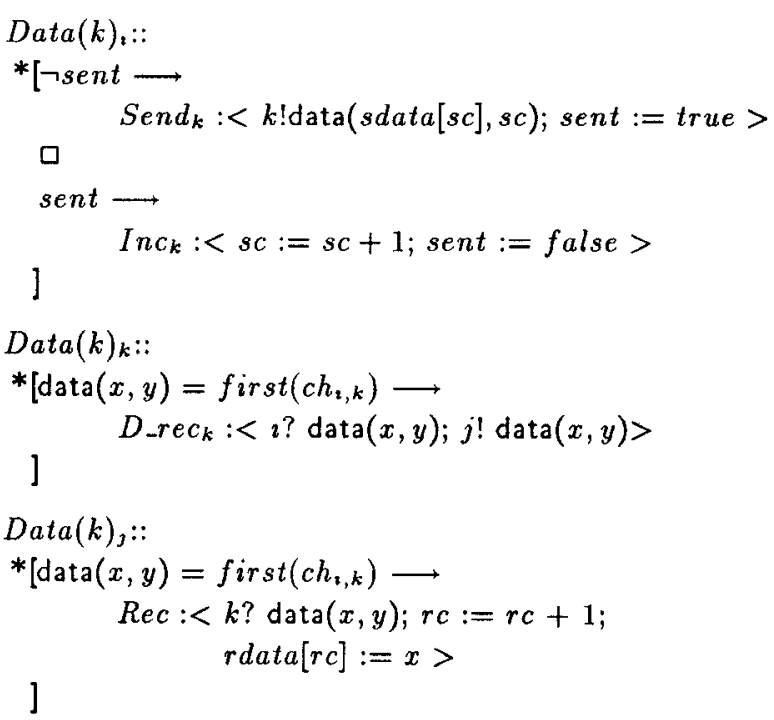

Figure 6: Data Transfer Protocol

receptions, (3) either $P$ dominates $Q$ or $Q$ dominates $P$.

For example, in the mutual exclusion protocol of Figure 5 obtained by composing $M 1$ and $M U T(j, k),\left(\right.$ Enter $_{j, k}$, Enter $\left._{k, j}\right)$ dominates $\left(\right.$ Enter $_{j, i}$, Enter $\left._{k, i}\right)$. Hence, MUT $(j, k)$ dominates $M 1$, and therefore, the resulting composite protocol is deadlock free. Note that checking condition (1)-(3) in Theorem 3.5 requires reachability analysis of the component protocols only. In the full paper, we give a more elaborate condition for deadlock detection. It involves constructing a graph whose nodes are element of $\operatorname{synch}(P, Q)$ and edges are obtained using the dominates relation. The acyclicity of this graph implies deadlock freedom.

\subsubsection{Liveness Properties}

We will give inference rules to derive liveness properties of $R$. In the following, we will assume that $R$ is free from unspecified assumptions. Following [LU90], en $\left(a_{p}\right)$ is interference-free if for all actions $b$ of $Q$, such that $b$ is not in $\operatorname{synch}(P, Q),\{e n(a) \wedge e n(b)\} b\{e n(a)\}$ is true (i.e., the execution of $b$ does not invalidate $e n\left(a_{p}\right)$ ). We will assume that all actions of $P$ and $Q$ are interferencefree.

Lemma 3.1 Let $\alpha \leadsto \beta$ via $a_{p}$ in $P$. If $a_{p}$ is not in $\operatorname{synch}(P, Q) \cup$ inhibit $(P, Q)$ then $\alpha \sim \beta$ เn $R$.

We say that $\alpha$ is stable, if in any execution, once $\alpha$ becomes true, it remains true. 


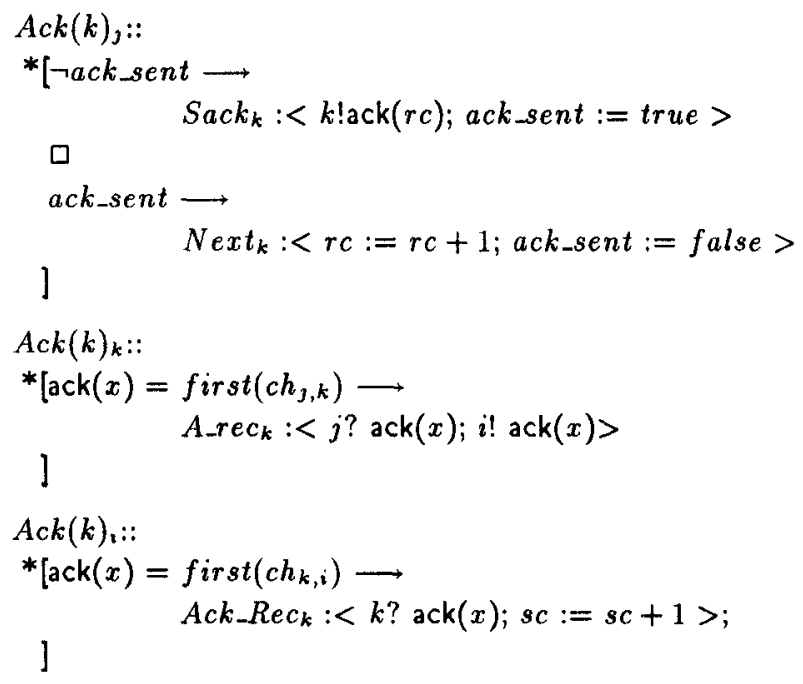

Figure 7: Acknowledgement Protocol

Lemma 3.2 Let $\alpha \leadsto \beta$ via $a_{p}$ in $P$. If $a_{p} \in$ inhibit $(P, Q)$ and $\beta$ is stable then $\alpha \leadsto \beta$ in $R$.

We say that $\alpha \sim \beta$ is a local property of $P$ if $\alpha \sim \beta$ in $P$ and in any execution $g_{0} \rightarrow e_{0} g_{1} \rightarrow g_{1} \cdots$ of $P$, for all $x$, if $\alpha$ is true in $g_{x}$ then $\beta$ is true in $g_{y}, y \geq x$, and $e_{l}$, $x \leq l \leq y$, does not belong to $\operatorname{synch}(P, Q)$ (informally, $\alpha \sim \beta$ is a local property if it can be inferred using rules (L1-L4) such that no application of L1 involves an action in $\operatorname{synch}(P, Q))$. Let $I$ be an invariant of $R$ and $\left(a_{p}, a_{q}\right) \in \operatorname{synch}(P, Q)$. We say that $a_{p}$ of $P$ is non_blocking if $\left(e n\left(a_{p}\right) \wedge I\right) \leadsto e n\left(a_{q}\right)$ is local to $Q$. We have the following theorem:

Lemma 3.3 Let $\alpha \sim \beta$ via $a_{p}$ in $P$. If $a_{p} \in$ synch $(P, Q)$ and each action in synch $(P, Q)$ is nonblocking then $\alpha \sim \beta$ in $R$.

For example, all actions of $M 1$ and $M U T(j, k)$ are interference free and all actions in $\operatorname{synch}(M 1, M U T(j, k))$ are non-blocking. We can show that $e^{x} t_{j} \sim i n_{j}$ in $\operatorname{MUT}(j, k)$. For each application of rule L1 in inferring this property, Lemma 3.1 or Lemma 3.3 applies. Hence, we can infer that $e x t_{j} \leadsto i n_{j}$ in $\operatorname{MUT}(i, j, k)$. Similarly, $\neg v_{j} \sim$ abort $_{i}$ in $A(i, j)$ and therefore, in $A 3 P(i, j, k)$, $\neg v_{j} \sim a b o r t_{i}$ will be true (from Lemma 3.2 ).

\section{A Data Transfer Protocol}

In this section, we will derive a data transfer protocol. Figure 6 gives a protocol $\operatorname{Data}(k)$ which transfers data item from $i$ to $j$ via node $k$. After sending a data item,

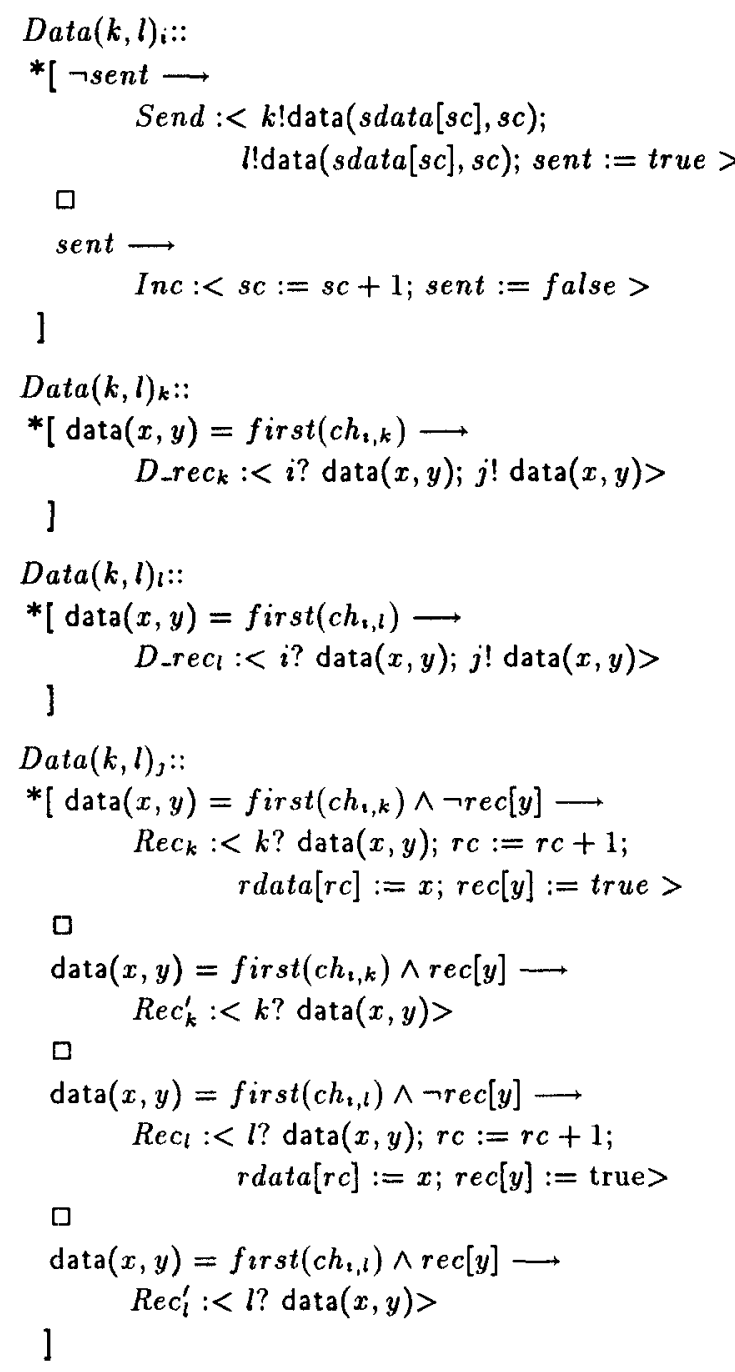

Figure 8: Combined Data Transfer Protocol

process $i$ increments a counter and then sends the next data item. On receiving the data item, $j$ increments a counter and stores the data item. Figure 7 gives a protocol $A c k(k)$ in which $j$ sends an acknowledgement to $i$ via $k$. In this protocol, $j$ sends acknowledgements to $i$ with increasing counter values. Process $i$ simply receives the acknowledgements. Similarly, we can obtain a protocol, Data(l) for transferring data from $i$ to $j$ via another site $l$. For reasons of fault-tolerance and efficiency, we may want to propagate the data via both $k$ and $l$. We can obtain this protocol by combining $\operatorname{Data}(k)$ and $\operatorname{Data}(l)$. We find that Data $(k)$ and Data $(l)$ are matching. We combine Data $(k)$ and $\operatorname{Data}(l)$ to obtain $\operatorname{Data}(k, l)$, shown in Figure 8, using: $\operatorname{synch}(\operatorname{Data}(k), \operatorname{Data}(l)=$

$\left\{\left(\right.\right.$ Send $_{k}$, Send $\left._{l}\right),\left(\right.$ Inc $_{k}$, Inc $\left.\left._{l}\right)\right\}$

$\operatorname{inhibit}\left(\operatorname{Data}(k), \operatorname{Data}(l)=\left\{\left(\operatorname{Rec}_{k}, \operatorname{Rec}_{l}\right)\right\}\right.$. 


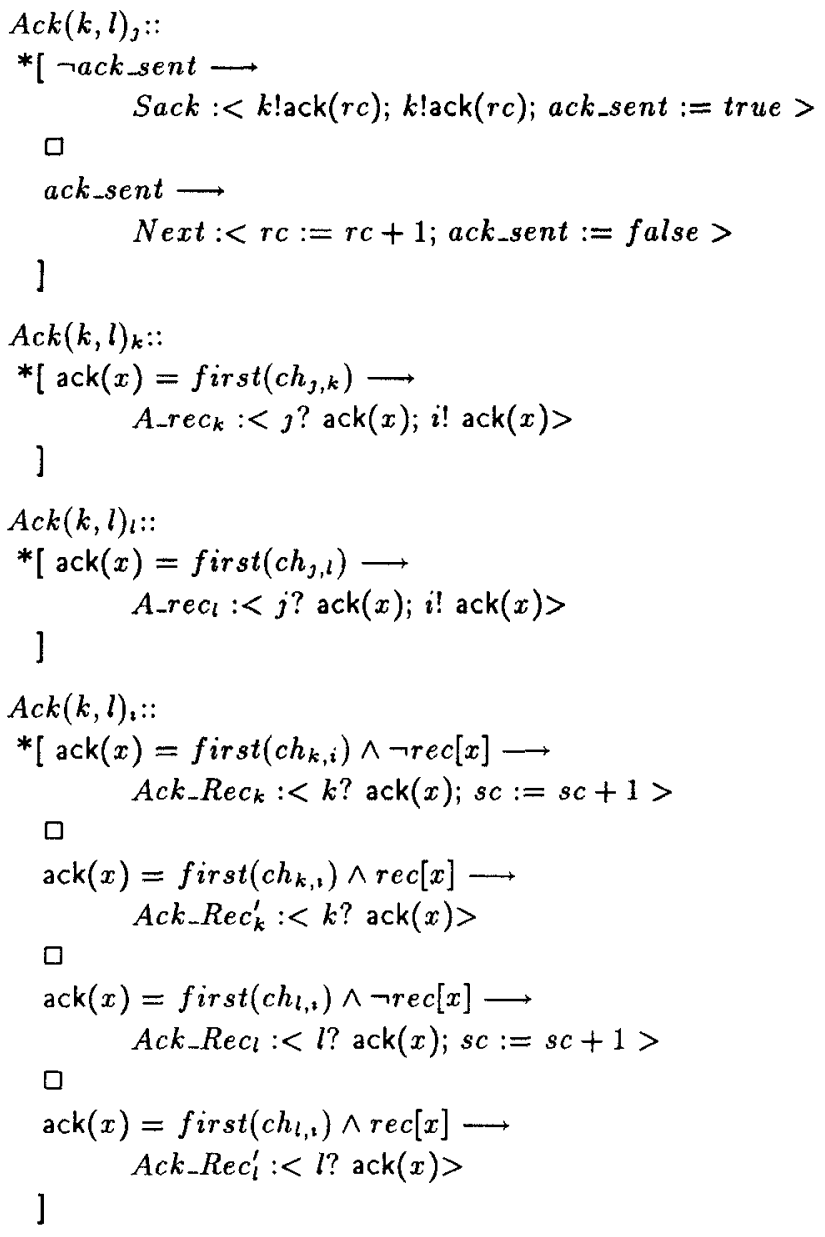

Figure 9: Combined Acknowledgement Protocol

In the composite protocol, Data $(k, l)$, process $j$ may receive the $x^{\text {th }}$ data item from either $k$ or $l$. It can be seen that the projection of an execution of $\operatorname{Data}(k, l)$ on to events of $\operatorname{Data}(k)$ may not be an execution of Data $(k)$. However, using the proof rules, we may still infer invariants of Data $(k, l)$ from those of $\operatorname{Data}(k)$. Similarly, $A c k(k)$ and $A c k(l)$ are matching and we combine them to obtain $A c k(k, l)$, shown in Figure 9, using

$\operatorname{synch}(A c k(k), A c k(l)=$

$\left\{\left(\operatorname{Sack}_{k}\right.\right.$, Sack $\left._{l}\right),\left(\right.$ Next $\left.\left._{k}, N e x t_{l}\right)\right\}$ $\operatorname{inhibit}\left(\operatorname{Ack}(k), \operatorname{Ack}(l)=\left\{\left(A_{c k} \operatorname{Rec}_{k}, A c k \_R e c_{l}\right)\right\}\right.$.

In the composite protocol, $A c k(k, l)$, the propagation of an ack for a data item takes place via both $k$ and $l$ and $i$ can receive it via either paths. We can verify that $\forall i<\operatorname{rc}($ sdata $[i]=\operatorname{rdata}[i])$ is an invariant of both $\operatorname{Data}(k)$ and Data $(l)$. Since it refers only to shared variables, it is also an invariant of $\operatorname{Data}(k, l)$ (from Theorem 3.2). Since $(s c>k) \sim(r c>k)$ in $\operatorname{Data}(k)$, and the protocol satisfy the preconditions of Lemma 3.2 (since $r c>k$ is stable), it is also a property of $\operatorname{Data}(k, l)$.

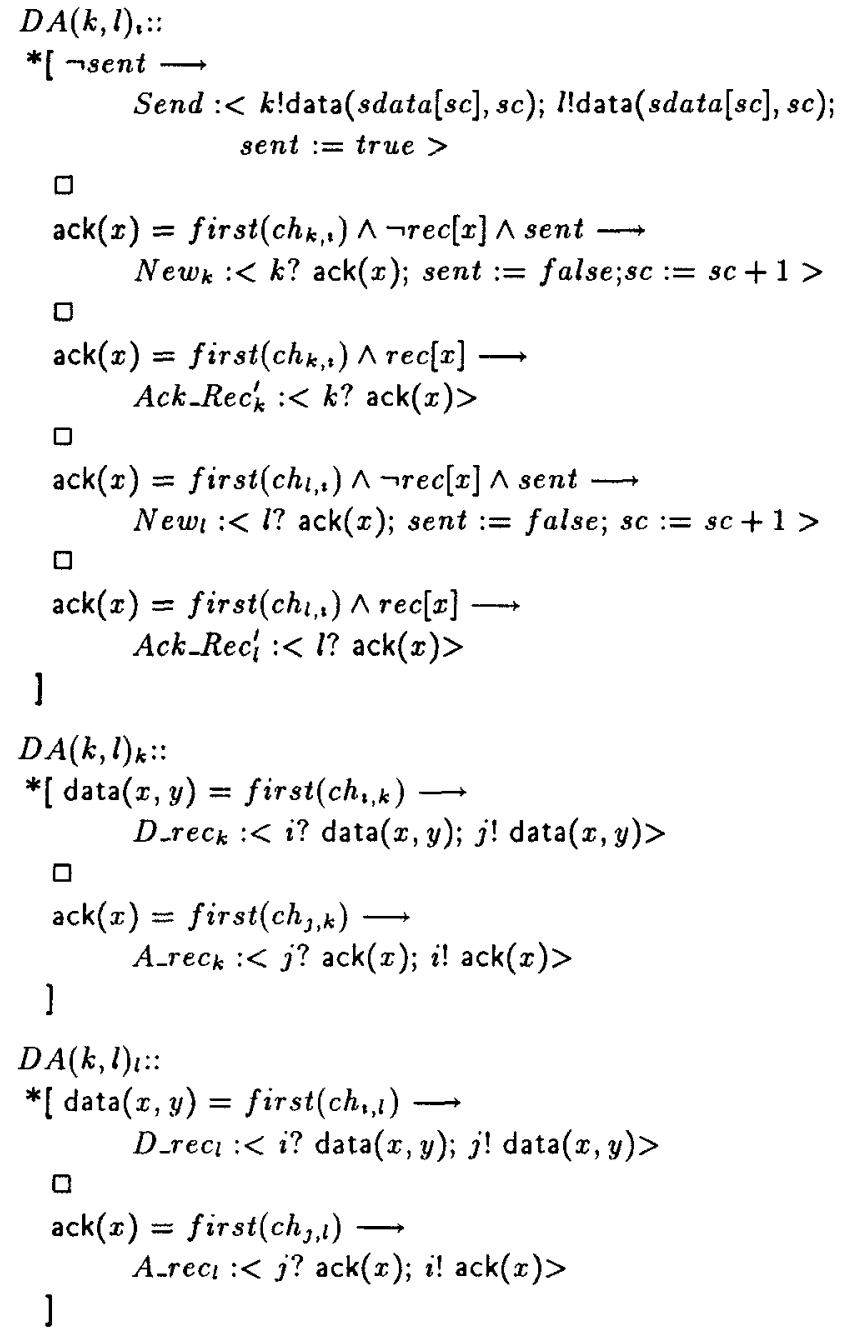

Figure 10: Combined Data and Acknowledgement Protocol (processes $i$ and $k$ )

Similar properties can be inferred for $A c k(k, l)$. We will now combine Data $(k, l)$ and $A c k(k, l)$ to obtain the final protocol, in which $i$ sends the next data item only on receiving an acknowledgment for the previous data item sent. The protocol, shown in Figure 10 and Figure 11, is obtained using

$\operatorname{synch}(\operatorname{Data}(k, l), \operatorname{Ack}(k, l))$

$\left\{\left(\operatorname{Rec}_{k}, N e x t\right),\left(\operatorname{Rec}_{l}, N e x t\right),\left(A c k-\operatorname{Rec} c_{k}, I n c\right)\right.$,

$(A c k-R e c l, I n c)\}$.

In the full paper, we show that the following are invariants of the final protocol:

$\forall i<\operatorname{rcsdata}[i]=\operatorname{rdata}[i])$;

$r c \leq s c \leq r c+1$ (using Theorem 3.3).

In the combined protocol, although $j$ may receive the $x^{t h}$ data item via $k$, process $i$ may receive the acknowledgement for this data item via $l$. 


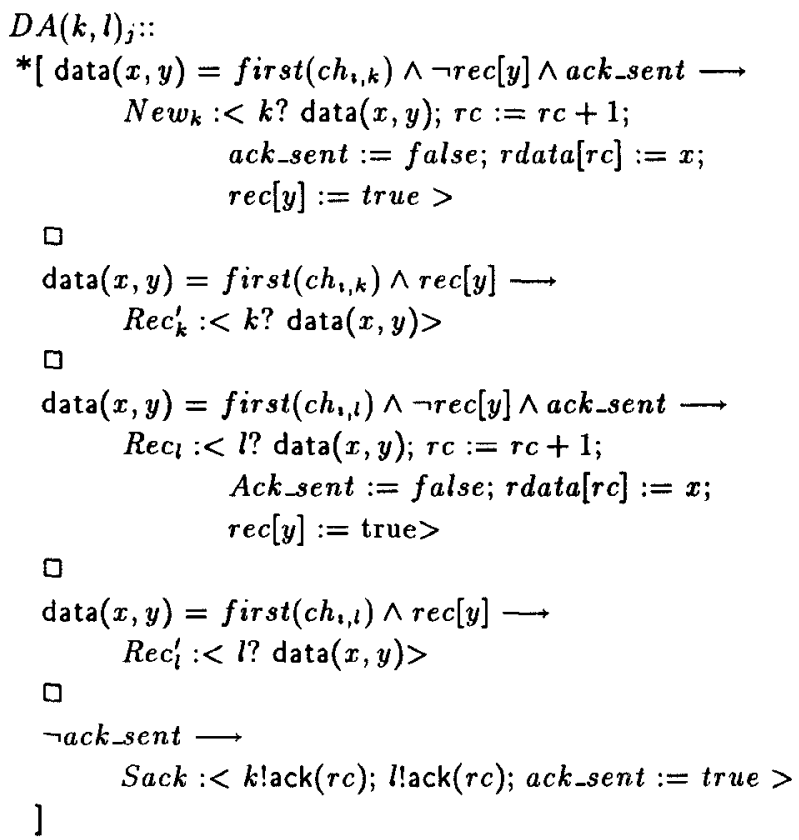

Figure 11: Combined Data and Acknowledgement Protocol(processes $l$ and $j$ )

\section{Conclusion}

In this paper, we have presented a technique for parallel composition of protocols. The protocols are combined by specifying the interaction between the events of the component protocols. We identified two different forms of interaction: The first involves synchronizing the actions of the component protocols and the other involves inhibiting actions in one protocol on the occurrence of certain action in the other protocol. We demonstrated the utility of our technique by deriving several protocols. We believe that our technique, in conjunction with sequential composition and step-wise refinement can be used to design and verify complex protocols.

\section{References}

[BF 88] Bouge, L. and Francez, N. A compositional approach to superimposition. In Proceedings of the ACM Symposium on Principles of Programming Languages, 1988.

[CGL85] Chow, C., Gouda, M., and Lam, S. A discipline for constructing multi-phase communicating protocols. ACM Transactions of Computer Systems, 3(4), 1985.
[CM83] Choi, T.Y. and Miller, R.E. A decomposition method for the analysis and design of finite state protocols. In Proceedings of the 8th Data Communication Symposium, 1983.

[CM88] Chandy, M. and Misra, J. Parallel program design. Addison-Weslay, 1988.

[FLS87] Fekete, A., Lynch, N., and Shrira, L. A modular proof of correctness for a network synchronizer. In Proceedings of the International Workshop on Distributed Algorithms, 1987.

[Jon94] Jonsson, B. Compositional specification and verification of distributed systems. ACM Transactions on Programming Languages and Systems, 16(2), 1994.

[Lin88] Lin, H. A methodology for constructing communication protocols with multiple concurrent functions. Distributed Computing, 3, 1988.

[Lin91] Lin, H. Constructing protocols with alternative functions. IEEE Transactions on Computers, 40(4), 1991.

[LT87] Lynch, N. and Tuttle, M. Hierarchical correctness proofs of distributed algorithms. In Proceedings of the ACM Symposium on Principles of Distributed Computing, 1987.

[LT93] Lin, H. and Tarng, C. An improved method for constructing multiphase communications protocols. IEEE Transactions on Computers, 42(1), 1993

[LU90] Lam, S. and Udaya Shankar, A. A relational notation for state transition systems. IEEE Transactions on Software Engineering, SE-16(7), July 1990.

[Sin93] Singh, G. A compositional approach for designing protocols. In Proceedings of the IEEE Int'l Conference on Network Protocols, 1993.

[SR89] Stomp, F. and Roever, W. de. Designing distributed algorithms by means of formal sequentially phased reasoning. In Proceedings of the 3rd Int'l Workshop on Distributed Algorithms, 1989.

[WLL88] Welch, J., Lynch, N., and Lamport, L. A lattice-structured proof technique applied to a minimum spanning tree algorithm. In Proceedings of the ACM Symposium on Distributed Computing, 1988. 